\title{
Researches of a WSN with Scale-free Routing Topology
}

\author{
Ye $\mathrm{Xu}^{1}$, Yun $\mathrm{Chi}^{2}$ and Xingyu Zhao ${ }^{1}$ \\ ${ }^{1}$ School of information science and engineering, Shenyang Ligong University \\ Shenyang, Liaoning 100159, China \\ ${ }^{2}$ Liaoning Academy of Governance, Shenyang, Liaoning 110161, China \\ xuy.mail@163.com,chiyun@21cn.com
}

\begin{abstract}
Based on the topological characteristics of scale-free networks, a kind of wireless sensor network routing algorithm based on scale-free was studied. We did our experiments from a perspective of the average degree distribution and the clustering properties of some high-degree nodes, or the "hot nodes" as we called them. First, we used multi-path principles to build a scale-free network topology based wireless sensor network and robustness is briefly discussed. Then, information fusion ability was introduced to balance network energy and extend the life cycle of the network, as a result of saving traffics. Simulations showed that the routing algorithm has more significant effect on the node degree distribution, reliability and overall performance evaluation once compared with some other WSNs.
\end{abstract}

Keywords: scale-free topology; wireless sensor network; routing algorithm; network life cycle

\section{Introduction}

Scale-free network model originated in 1999 by a paper entitled "emergence of scale in random networks"[1]. Scale-free characteristic of complex network, also called as Matthew's law in other fields was introduced. A very important property of Matthew's law in complex networks is that the degree of the majority of nodes is very low while that of very few nodes having very high degree. On the basis of this theory, Professor Barabasi has established a scale-free model, which is now referred to as the BA model.

The degree distribution obeys the power law distribution in scale-free networks. The properties of network growth and preferential attachment[1] are scale-free networks signature feature. Power exponent could have great affects on the uniformity of network, which as a result, has enormous influences on the robustness of networks. At present, topological structure characteristics and models of scale-free network are mainly studied, however, applications of the scale free theory to wireless sensor networks is relatively rare. In literature 2, studies were done on the preferential attachment of the network considering adsorption properties of the nodes in networks. A definition of the effective cost which equals the product of node strength of the path was put forward in literature 3 , connections between the packet queue length and delivering output were used to adjust the weight of the neighbor nodes so as to improve network throughput. In literature 4 , a way to improve scalability of the network through better balancing the relationship between the routing table size and elongation factor out of studies of properties of power law and the strong aggregation of scale-free network. And in literature 5, studies on network traffic model and node betweeness were studied to improve network capacity of the model. Literature 6 a dynamic local routing was given based on cross studies between physical characteristics of scale-free networks and network node queue length. 
In literature 7, influences on routing networks by network topology and the processing speed of the nodes was studied. Method of parameter optimization of both static and dynamic models were put forward. The literatures mentioned above give different studies from diverse point of view, however, topics on routing WSN from complex networks properties are rarely studied.

In this paper, we'll focus on a scale-free routing algorithm, named as SFRA, designed by properties of scale-free topology in complex networks, and topics such as data transmission, network energy consumption, surviving nodes number and the network life cycle are mostly covered.

\section{Scale Free Network Model}

Properties of network growth and preferential attachment are the two keys while constructing a scale free network topology. And due to these characteristics, we can give an explanation why scale-free networks tend to have power-law distribution. Therefore, scalefree networks can be also called as the network with a power-law distribution in nodes' degree.

With these properties, we could try to establish a certain preferential attachment mechanism in wireless sensor networks, which might be able to balance the energy distribution of sensor nodes, thereby extend the life cycle of network. Besides, network topology could also be controlled to result in uniformities in the network, which in return, would result in a good capability of robustness against random and target attacks[9][10]. Since the wireless sensor network with scale-free properties is a network having a major of nodes with rather low degree but a small number of nodes with very high degree, it is important to study the energy consumption of nodes with high degree in extending life cycle point of view.

Scale-free network is different from regular network and small-world network. It's a kind of complex network constructed to be a topology between regular networks and random networks by certain policies.

\subsection{Scale-free Network Properties}

Some of the basic definitions of the scale-free network are as follows:

Definition 1: assuming that undirected graph $\mathrm{G}=(\mathrm{V}, \mathrm{E}), \mathrm{N}=|\mathrm{V}|$ represents the number of nodes in the network, $M=|E|$ is the number of edges of the network. The degree of node $I$ is defined as: the number of nodes $I$ and the neighbor nodes connected, remember to do $\boldsymbol{k}_{\boldsymbol{i}}$.

Definition 2: Degree of a random node in network is called degree distribution. Node degree in Scale-free networks follows a power law distribution with function of $p(k)$, which means any node in the networks have a probability $p$ having $k$ edges. Then the relationship between the $p(k)$ and the size of node $k$ is:

$$
P(k) \propto k^{-\gamma}
$$

We can judge whether a network has power-law properties by equation (1).

Definition 3: The network with power-law distribution in nodes follows the law of Matthew. A majority of nodes have very low degree but very few nodes have very high degree. The nodes with high degrees are called hot nodes, while the others are called common nodes.

Definition 4: During a network growth, once a new node is added to the networks, there is a high probability that it has priority to connect some kind of nodes with certain properties, i.e., nodes having high degree. And this is called preferential attachment in network topology growth. 
Definition 5: Clustering coefficient gives definition of the relations between one node and its neighbors in the network, and it also shows how much nodes could aggregate or closely connected to each other in the network:

$$
C_{i}=\frac{2 E_{i}}{k_{i}\left(k_{i}-1\right)}
$$

In which, $k_{i}$ is the degree of node $I, E_{i}$ is the number of edges between $k_{i}$ nodes and node $I$, and $\frac{k_{i}\left(k_{i}-1\right)}{2}$ represents the total number of possible edges between $k_{\mathrm{i}}$ nodes ${ }^{[9]}$.

Definition 6: A small size network consisting a hot node and other neighbor nodes in the network is defined to be a cluster in the network, and the hot node in this cluster is called the head $(\mathrm{CH})^{[10]}$. Nodes that are connected to all these heads are called the sink nodes in the networks.

Definition 7: The scale free network is highly robust against random attacks, and is vulnerable during a deliberate or target attack.

\subsection{Network Topology Model}

Wireless sensor networks based on scale-free properties are constructed by the connection of common nodes, hot nodes and sink nodes. Hot nodes and common nodes form clusters. Several hot nodes could form an upper layer of cluster by connecting to each other, and so on until all cluster heads connect to the sink node. In this view, the networks is divided into different layer of clusters connected to each other through cluster heads or hot nodes.

Common nodes function to receive, store and transmit messages, while hot nodes function to receive messages from the common node. The messages would be routed and transmitted to upper layer of hot nodes or sink node (once in the last layer of clusters) after message handling by information fusion so as to save energies.

In the initial state, the network contains $\boldsymbol{m}_{\boldsymbol{0}}$ isolated nodes.

(1) Growth Characteristics: During each time period, a new node will be added to the network and connect with other nodes in the network.

(2) Preferential Attachment: Assuming the node I has self adsorption index A, then the probability of the newly added nodes connected to node $I$ is $\Pi_{i}$, then:

$$
\Pi\left(k_{i}\right)=\frac{k_{i}+A}{\sum_{j}\left(k_{j}+A\right)}
$$

After time period $\boldsymbol{t}$, we could get the network with nodes $\boldsymbol{N}=\boldsymbol{t}+\boldsymbol{m}_{\boldsymbol{0}}$ and edges $\boldsymbol{m} \boldsymbol{t}$.

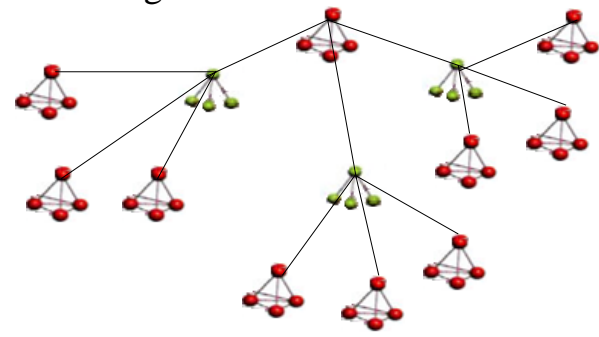

\section{Figure 1. Topology of Wireless Sensor Network with Scale-free Properties}

As is shown in figure 1, common nodes are connected to the hot node at the bottom layer. The hot nodes of bottom layer, however, connect to each other to form the upper layer, and eventually reach the sink node. During topology construction period, the newly added common nodes would try to connect to certain nodes by degree and adsorption index of hot node according to preferential attachment properties, and send the connection messages to the hot node. After the hot node 
receives all the messages, it will generate a TDMA signal and notify all the common nodes. Then the common node stores the cluster header information of its cluster. This procedure repeats till all nodes are added and the connections between the cluster header and common nodes is established. Some of the newly added common nodes, of course, would be transferred into hot nodes during constructions.

\section{Mathematical Analyses}

We try to solve the node degree distribution in the wireless sensor networks by means of continuous domain theory ${ }^{[11]}$. Assume a new node $I$ joins the network with $\boldsymbol{m}_{0}+t$ nodes at the time $t$, and the degree $\boldsymbol{k}_{\boldsymbol{i}}$ of node $I$ is a continuous variable. Then the rate of continuous change rate of $\boldsymbol{k}_{\boldsymbol{i}}$ is:

$$
\frac{\partial k_{i}}{\partial t}=m \cdot \pi\left(k_{i}\right)=m \cdot \frac{k_{i}+A}{\sum_{j}\left(k_{j}+A\right)}
$$

At $T$ time, the total network node degree is:

$$
\sum_{j}\left(k_{j}+A\right)=2 m t
$$

Substitute equation (5) into (4), we get the simplified equation:

$$
\frac{\partial k_{i}}{\partial t}=m \cdot \frac{k_{i}+A}{\sum_{j}\left(k_{j}+A\right)}=m \cdot \frac{k_{i}+A}{2 m t}=\frac{k_{i}+A}{2 t}
$$

Solving differential equations, and substituting the initial conditions $k_{i}\left(t_{i}\right)=m$. After calculation and simplification, we get:

$$
k_{i}(t)=(m+A)\left(\frac{t}{t_{i}}\right)^{\frac{1}{2}}-A
$$

Then the probability expression of node $\boldsymbol{I}$ degree less than $\boldsymbol{k}$ is:

$$
p\left(k_{i}(t)<k\right)=p\left(\left((m+A)\left(\frac{t}{t_{i}}\right)^{\frac{1}{2}}-A\right)<k\right)=p\left(t_{i}>t\left(\frac{m+A}{k+A}\right)^{2}\right)=1-p\left(t_{i} \leq t\left(\frac{m+A}{k+A}\right)^{2}\right)
$$

Since only one node is added into the networks each time period, we take for granted that $\boldsymbol{t}_{\boldsymbol{i}}$ follows an uniform distribution and its probability density function is $p\left(t_{i}\right)=\frac{1}{m_{0}+t}$. After substitution we get equation:

$$
p\left(k_{i}<k\right)=1-\left(\frac{m+A}{k+A}\right)^{2} \frac{t}{m_{0}+t}
$$

And it would be simplified into:

$$
p(k)=\frac{\partial p\left(k_{i}(t)<k\right)}{\partial k}=\lim _{t \rightarrow \infty} \frac{t}{m_{0}+t} \cdot 2(m+A)^{2}(k+A)^{-3}=2(m+A)^{2}(k+A)^{-3}
$$

From the above we could find that the node degree distribution of the wireless sensor network follows a power law distribution of $2(m+A)^{2}(k+A)^{-3}$, which could be a proof that the constructed wireless sensor networks has properties of scale-free networks.

The above analyses that the degree distribution of wireless sensor network follows the power law distribution are primary knowledge for our next works. 


\section{Routing Algorithm Design}

\subsection{SFRA Routing}

The procedure of scale-free wireless sensor network routing algorithm (SFRA) is shown in figure 2.

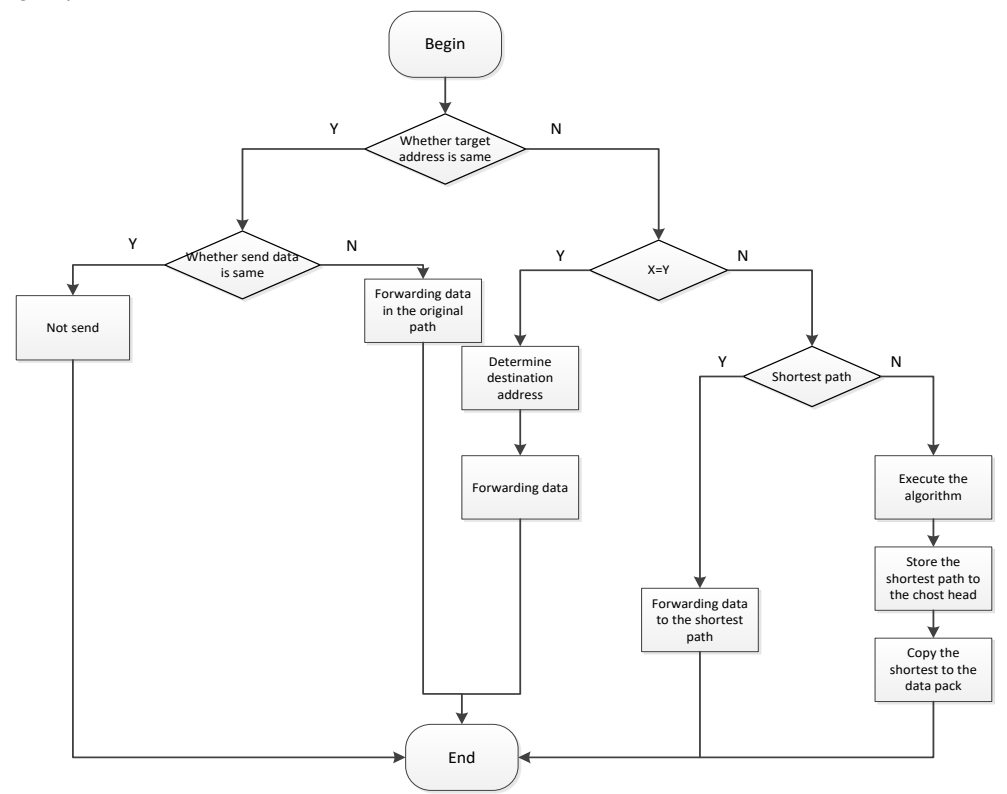

Figure 2. Flowchart of SFRA Algorithm

And the algorithm is as follows:

(1) Waiting for message. Assuming node A send message to node B. First, a test whether the target address equals to that stored during last step. If equals turn to (2), otherwise turn to (3);

(2) Further test whether the message is same to that stored during the last step. If so, the message is discarded. Otherwise, algorithm forwards the message using the last routing path after searching the routing table.

(3) Assuming $\mathrm{CH}$ of cluster where node $\mathrm{A}$ is located is $X$, the $\mathrm{CH}$ of cluster where node B is located is $Y$, a test whether $X$ equals $Y$ is performed. If $X=Y$ turn to (4), otherwise turn to step (5);

(4) Since $X=Y$, the communication is within a cluster. The algorithm directly forward data according to the shortest path after setting the target address and obtaining the ID number of target node;

(5) To determine whether there is the shortest path in routing table. If so, the message is forwarded. Otherwise turn to (6);

(6) $\mathrm{CH} X$ transmits the message to the $\mathrm{CH} Y$. Algorithm regards the wireless sensor network as an undirected graph, CDZ algorithm ${ }^{[12]}$ is and implemented by $\mathrm{CH} X$ to calculate the shortest path to forward message, then to store it to the routing table of cluster head cache and to inform all the related nodes in this path ${ }^{[14]}$. After this procedure, the message is transmitted by the shortest path in routing table from $\mathrm{CH} X$ to $\mathrm{CH} Y$. $\mathrm{CH} Y$ sends the data to node B since they are in the same cluster. And turn back to (1).

\subsection{Routing Robustness Analysis}

Robustness refers to the properties that when a node dies due to energy exhaustion or attacks in WSN, the remained nodes could still be capable of 
maintaining normal communication, topology and performance of the network. The routing robustness ${ }^{[15]}$ is the ability to properly route and maintain overall performance to an acceptable level in the networks no matter what uncontrollable external factors there would be. Therefore, routing robustness is an important basis to evaluate the performance of a routing algorithm.

SFRA routing algorithm is dependent on the scale free topology of the target wireless sensor networks. As mentioned above, the node degree distribution of the scale free network obeys a power law property. And the wireless sensor networks with such features thus have an excellent robustness ${ }^{[16]}$ against random attacks. However, the hot nodes in the networks are relatively vulnerable to target attacks ${ }^{[16]}$, and the failure of the hot nodes will effectively influence the communication quality of the wireless sensor network.

(1) Robustness of common nodes

When the common nodes fails due to energy depletion or attacks, the node will send a message to notify hot node of the cluster before the expiry. After receiving the message, the hot node will update the routing table according to the current situation in the cluster. Such cases would have little influence on the overall robustness performances of the networks.

(2) Robustness of hot nodes

Since the hot node usually has large degree, their energy consumption is much higher than others. Once the energy of the hot node is lower than the set threshold or being attacked, the communication and robustness performance of the entire cluster will be affected. In the algorithm, the dying hot node will send message to upper layer cluster head before the failures (reaching the threshold). When nearly half of the cluster head in the network fail, the algorithm would set that the sink node be notified. Then the sink node broadcast reconstruction message to all hot nodes and all common nodes by transfer of hot nodes. And SFRA routing algorithm would re construct the entire networks and routing tables.

\section{The Simulation Analysis}

\subsection{Simulation Environment}

The experiment area is $200 \mathrm{~m} \times 200 \mathrm{~m}$ square large. About 2000 wireless sensor network nodes are set in the networks and the base coordinates is $(0,0)$. The position of the sensor node is fixed according to this base coordinate. Sensing range of the nodes is $30 \mathrm{~m}$.

Simulation experiment: CPU is Intel Core $\mathrm{i} 3$, memory $2 \mathrm{G}$, the operating system is Windows 7, and simulation software is MATLAB.

\subsection{Algorithm Performance Index}

In order to evaluate the performance of SFRA routing algorithm, we did experiments considering properties of network topology, node degree distribution, message transmission, energy consumption, lifecycle of network and robustness. Parameter of message transmission is important in measuring lifecycle of the networks because message transmissions cost lots of energy ${ }^{[4-6]}$. For saving more energy, the algorithm would transmit messages through the shortest routing path and the shortest time.

Network life cycle is the survival time of the network, in other words, the network is still alive when there is still a large connected subgraph enough for maintain basic communications. And parameter robustness gives monitor of such properties in experiments. 
In simulation experiments, we give contrasts between the SFRA and LEACH in different experiments indexes and parameters mentioned above.

\subsection{Simulation Experiments 1: Data Analyses}

Figure 3 gives the topology of wireless sensor networks. And the related parameters are: set initial network node number $m_{0}=5$, new generation edges number when introduced new nodes $m=5$, network size $N=130$. After the experiment, the clustering coefficient of network graph is 0.15 , and the average path length is 2.35 .

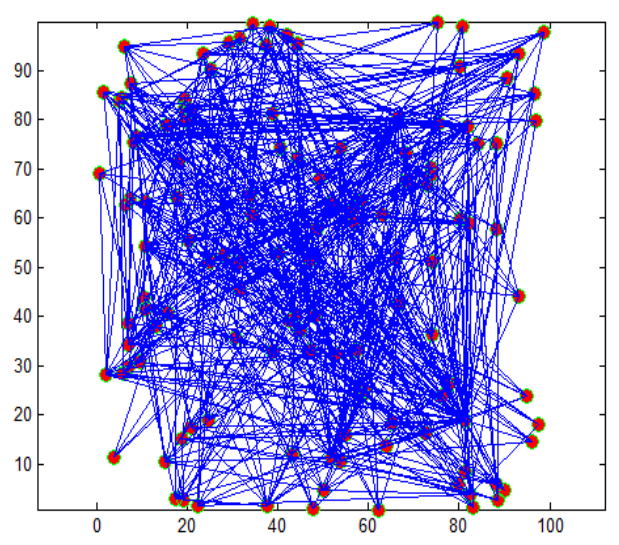

Figure 3. WSN Topology Diagram

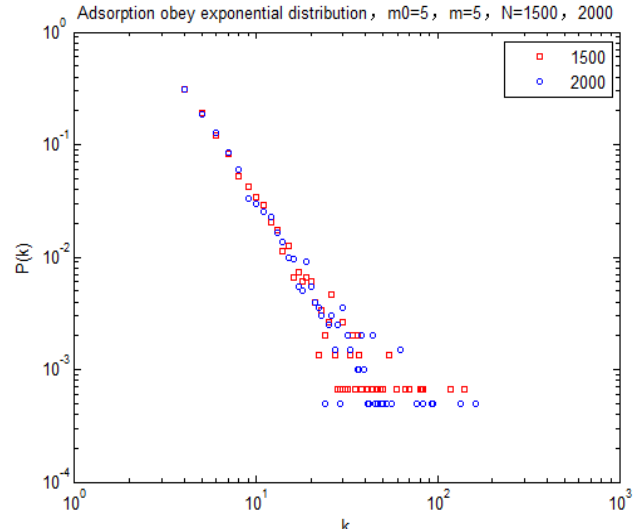

Figure 4. WSN Degree Distribution Diagram

Figure 4 gives the degree distribution of wireless sensor networks. We did experiments on the different networks with size $N=1500$ and $N=2000$ respectively. As can be seen from the figure, degree distribution of sensor nodes obeys the power law property. The correctness of the theory could be verified.

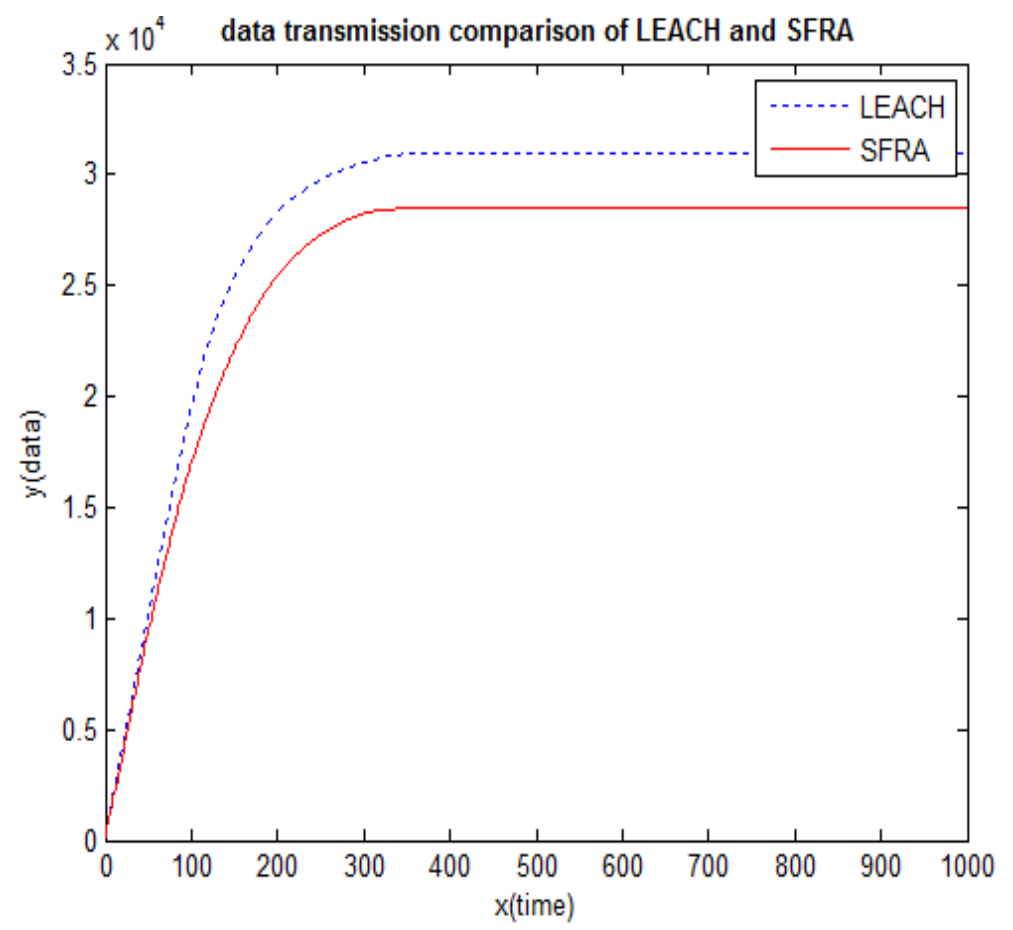

Figure 5. Message Transmission Comparison between LEACH and SFRA 
Figure 5 gives message transmission performance comparison results. It can be seen from the figure that the transmission efficiency of SFRA is higher than that of LEACH. Since LEACH must communicate and forward data with the cluster head, but the cluster head storage space is limited, the data in unit time is also limited. Relatively, the SFRA algorithm has the use of the shortest path algorithm where routing path is stored, so that amount of transmitted data is less than LEACH.

Figure 6 reflects the energy consumption of the two algorithms. From the diagram, it's easy to find that the energy consumption growth rate of SFRA is less than LEACH, and the lifecycle is longer under the same workloads. At first period of time, the energy consumption of the two algorithms is basically identical. SFRA, however, is slightly better in energy performance than that of SFRA when time increases. Like what's seen in Figure 5, SFRA is slightly better than LEACH in energy consumptions due to the scale free shortest routing path algorithms. Since SFRA still has to re-select cluster head and random amount of the message in simulation experiments has to be transmitted between nodes far away from each other and the energy consumption is inevitable, there is no significant advantage of SFRA over LEACH in simulations. Given a specific circumstance that long distance transmission is rare, SFRA would show its advantages in energy saving aspect.

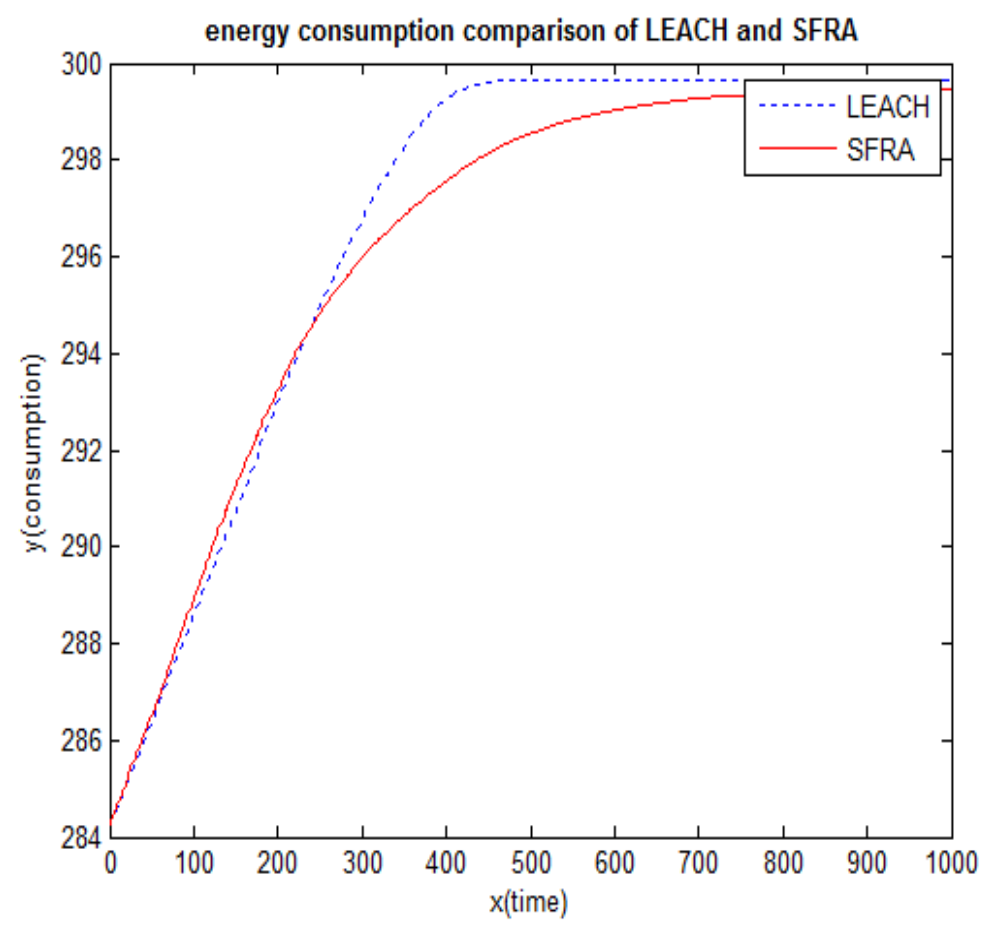

Figure 6. Energy Consumption Comparison Chart of LEACH and SFRA 


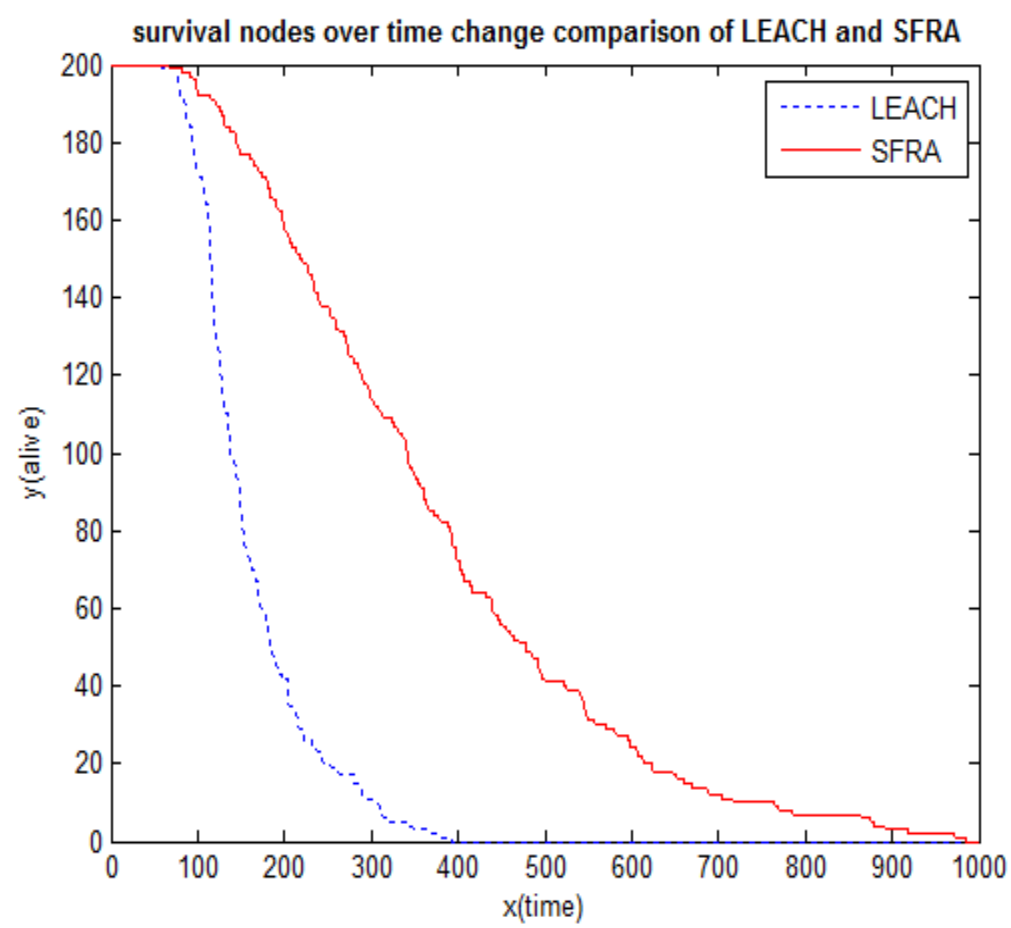

Figure 7. Survival Number of Nodes with Time in LEACH and SFRA

Survival nodes or remained nodes are important factors measuring performance of target algorithm and corresponding experiments are performed and illustrated in Figure. 7.

It can be seen from the figure that SFRA is superior to LEACH in remained survival nodes. Number of survival nodes in both algorithm drops sharply during simulations, and due to shortest routing algorithm, SFRA would save certain amount of energy during message delivery and remain to survive a little longer than LEACH. Besides, LEACH does not considered the distribution of cluster head at the time of election. Energy consumption of nodes in this area is large when the elected cluster heads are close to each other.

\subsection{Simulation Experiments 2: Performance Evaluations}

We mainly focus on comparison among SFRA, Flooding algorithm, LEACH algorithm and NRBBRP algorithm[13].

(1) Overall performance evaluations

\section{Table 1. Overall Performance Evaluation of Four Routing Algorithms}

\begin{tabular}{|c|c|c|c|c|}
\hline Algorithm & SFRA & Flooding & LEACH & NBEERP \\
\hline WSN life cycle & long & short & middle & middle \\
\hline $\begin{array}{l}\text { Computational } \\
\text { complexity }\end{array}$ & high & low & middle & middle \\
\hline $\begin{array}{l}\text { Implementation } \\
\text { complexity }\end{array}$ & middle & low & middle & high \\
\hline $\begin{array}{c}\text { Logical } \\
\text { topology } \\
\text { structure }\end{array}$ & hierarchical & horizontal & hierarchical & hierarchical \\
\hline
\end{tabular}


Flooding algorithm is a kind of flat routing algorithm mainly using the broadcast method to select path, and has a comparative low computational complexity. LEACH algorithm, SFRA algorithm and NEBBRP algorithm have higher computational complexity due to their hierarchical routing architecture. The SFRA algorithm is almost as much as the LEACH algorithm in computational complexity.

(2) Routing reliability evaluations

Table 2. Reliability Evaluation of Four Routing Algorithm

\begin{tabular}{|c|c|c|c|c|c|c|}
\hline \multirow[t]{2}{*}{ Algorithm } & \multicolumn{5}{|c|}{ Simulation Time ms } & \multirow{2}{*}{$\begin{array}{c}\text { Average } \\
\text { reliability\% }\end{array}$} \\
\hline & 20 & 40 & 60 & 80 & 100 & \\
\hline Flooding & 89.01 & 86.23 & 75.65 & 67.71 & 58.13 & 75.35 \\
\hline LEACH & 70.21 & 67.77 & 69.38 & 64.82 & 56.68 & 65.77 \\
\hline NBEERP & 83.22 & 93.35 & 89.49 & 85.80 & 71.76 & 84.52 \\
\hline SFRA & 90.30 & 93.21 & 96.66 & 95.86 & 93.50 & 93.91 \\
\hline
\end{tabular}

In experiments, the message correctly received by the sink nodes within the given period of time in all four algorithms is used to judge the reliability. The more messages received correctly in less time, the more reliable an algorithm is. SFRA algorithm uses information fusion to reduce the message traffic and increase the reliability compared with other algorithms.

(3) Evaluations of network node degree distribution

Due to the power law properties of scale free topology, the SFRA algorithm has a variable power exponent. As is well known, the power exponent is closely related to performance of networks, an increase of power exponent would result in high efficiency in communications and low robustness against target attacks. So the SFRA algorithm could be easily adjusted to different environment for specific requirements.

The node degree distribution of network with Flooding algorithm is uniform distribution with average degree is 6 . LEACH algorithm does not have prescriptive degree distribution, and the network topology is also relatively random, the average connection degree is 4 .

Table 3. Node Degree Distribution of Four Routing Algorithms

\begin{tabular}{|c|c|c|c|}
\hline Algorithm & Degree distribution & $\begin{array}{c}\text { Average } \\
\text { connectivity }\end{array}$ & $\begin{array}{l}\text { Network topology } \\
\text { structure type }\end{array}$ \\
\hline Flooding & Uniform distribution & 6 & Regular network \\
\hline LEACH & $\begin{array}{l}\text { Nonuniform } \\
\text { distribution }\end{array}$ & 4 & Random network \\
\hline NBEERP & Cubic distribution & 3.8 & No classification \\
\hline SFRA & $\begin{array}{l}\text { Power law } \\
\text { distribution }\end{array}$ & $\begin{array}{l}\text { Variable(greater } \\
\text { than 2) }\end{array}$ & Scale free network \\
\hline
\end{tabular}

From the above analyses, we see that the SFRA algorithm has advantages over others in aspects of the overall evaluation, routing reliability. And node degree distribution of SFRA is flexible according to specific requirements. By the experiments, the algorithm could be useful in routing wireless sensor network with limited energy and dynamic topology. 


\section{Conclusions}

The wireless sensor network routing algorithm based on the scale free topology properties were studied in this paper. For a better overall performance of the networks, scale-free specific topology structure is generated into WSN and the CDZ algorithm is introduced to calculate the shortest path of message delivery. Simulations experiments showed that the proposed algorithm had a great advantage in reducing the energy consumption of sensor networks, and extending the network life cycle than the others given routing algorithm. Adjustment of power exponent would help SFRA to the flexible applications in different environment requirements. The networks would show shorter routing path but lower robustness against target attacks with higher power exponent. And a quantitative research of power exponent adjustments would be out next work.

\section{Acknowledgement}

This work is financially supported by the National Natural Science Foundation of China (No. 61373159), the program for Liaoning Excellent Talents in University (LJQ2015095), the Shenyang Key Laboratory Project and the Open foundation of Key lab of Information Networking and Confrontation of Shenyang Ligong University (No. 4771004kfs18).

\section{References}

[1] Barabási A L, Albert R. Emergence of scaling in random networks[J]. Science, 1999, 286(5439): 509512.

[2] Shen Chaojie.Evaluation of the degree distribution of the Improved BA complex network model[D]. Hebei University of Technology,2007.

[3] Guo Wenzhong,Liu Zhanghui,Wang Xiaoxi,etc.A hybrid routing algorithm with adaptive coefficients in the scale-free network with power[J]. Mini Computer System,2014,3:4.

[4] Tian Yong,Tang Zhenan, Yu Yan.An adaptive clustering routing algorithm for indoor wireless sensor networks with energy balance[J]. Journal of Electronics and Information Science, 2013, 35(12):29922998.

[5] Zhu Zhinan,Zhu Lei,Chen Jianbin.An improved scale-free network model[J]. Military Communication Technology.2010,2:8

[6] Wen Hong,Fan Xiaoping,Zhang Huifu,etc.Design of dynamic local routing policy o the scale free network[J]. Computer Engineering and Applications, 2014, 50(20): 10-14.

[7] Wen Hong,Fan Xiaoping,Zhang Huifu,et al.Optimization and design of local routing algorithm for scale free network[J].Journal of Hunan University Natural:Science editon, 2014, 41(10): 122-128.

[8] Zhou Xiancun,Li Mingxi,Chen Zhenwei,etc.Based on hybrid hierarchical,probabilistic packet markers of WSNs node localization algorithm[J].Journal of Electronics and Information Science, 2014, 36(2): 384-389.

[9] Wang Xiaofan,Li Xiang,Chen Guanrong.Complex network theory and its application[M].Tsinghua University Press Limited Company, 2006

[10] Xu J, Wang X F. Cascading failures in scale-free coupled map lattices[J]. Physica A: Statistical Mechanics and its Applications, 2005, 349(3): 685-692.

[11] Han Tao. Research on WSNs topology control algorithm based on the theory of scale free[D].Yan Shan University, 2014.

[12] Ding Nan,Tan Guozhen,You Di,etc.An opportunity routing algorithm based on WSN time variant and energy balance of nodes remaining energy balance[J], 2013, 35(3): 715-720.

[13] Su Jiwei,Zhao Hai,Zhang Wenbo,etc.A wireless sensor network routing algorithm based on embedded technology[J].Computer Engineering, 2006, 32(14): 87-88.

[14] Tang F, You I, Guo S, et al. A chain-cluster based routing algorithm for wireless sensor networks[J]. journal of intelligent manufacturing, 2012, 23(4): 1305-1313.

[15] Ye F, Zhong G, Lu S, et al. A robust data delivery protocol for large scale sensor networks[C]//Information Processing in Sensor Networks. Springer Berlin Heidelberg, 2003: 658-673.

[16] Kollios G, Byers J W, Considine J, et al. Robust Aggregation in Sensor Networks[J]. IEEE Data Eng. Bull., 2005, 28(1): 26-32. 


\section{Authors}

Ye XU (1976-), received his ph.D degree in major of computer application technology in 2006 from Noreastern University, China. $\mathrm{He}$ is now working as a full professor in Shenyang Ligong University, China. And his research interests now include Network science, bigdata and Intelligent Systems. 